\title{
Physicians' Attitudes on Management of Osteopenia in South Korea
}

\author{
Joo Hyung Ha*, Hong Seok Kim*, Samuel Jaeyoon Won, Young-Kyun Lee, Kyung-Hoi Koo \\ Department of Orthopaedic Surgery, Seoul National University Bundang Hospital, Seoul National University College of Medicine, \\ Seongnam, Korea
}

Corresponding author

Young-Kyun Lee

Department of Orthopaedic Surgery, Seoul

National University Bundang Hospital, Seoul

National University College of Medicine,

82 Gumi-ro 173beon-gil, Bundang-gu,

Seongnam 13620, Korea

Tel: +82-31-787-7204

Fax: +82-31-787-4056

E-mail: ykleemd@gmail.com

Received: July 9, 2020

Revised: August 5, 2020

Accepted: August 6, 2020

*Joo Hyung Ha and Hong Seok Kim contributed equally to this work and should be considered cofirst authors.
Background: Osteopenia patients have a risk of fracture and may develop osteoporosis. We investigated physicians' management of osteopenia patients in South Korea.

Methods: A survey was conducted using a questionnaire including 6 items: (1) do you think anti-osteoporosis medications are necessary in osteopenia patients?; (2) what is your preference to manage osteopenia patients, except for anti-osteoporosis medications?; (3) what is your indication for the anti-osteoporosis medication in osteopenia patients?; (4) what kind of anti-osteoporosis medication do you prefer to treat osteopenia patients?; (5) do you use bisphosphonates?; and (6) if not, what is the reason for not using?.

Results: Among the 173 participants, 150 (86.7\%) replied that anti-osteoporosis medications were necessary in osteopenia patients. Indications for the medication were (1) past medical history of pathologic fracture in 85 (49.1\%); (2) T-score $<-2.5$ on dual energy X-ray absorptiometry in 73 (42.2\%); (3) previous history of osteoporosis in 44 (25.4\%); (4) risk of fracture according to fracture risk assessment tool in 34 (19.7\%); and (5) progressive bone loss in 31 (17.9\%). One hundred and sixteen (67.1\%) favored bisphosphonates, 93 (53.8\%) selective estrogen-receptor modulator, and 24 (13.9\%) hormone replacement therapy. Thirty-one (17.9\%) replied that they do not use bisphosphonates due to (1) restricted reimbursement by the health insurance in 24 (77.4\%); and (2) bisphosphonate-related complications in 19 (61.3\%).

Conclusions: Most respondents (86.7\%) thought anti-osteoporosis medications were necessary in osteopenia patients, but $17.9 \%$ of the respondents did not use bisphosphonates. Restricted reimbursement by the national health insurance was the major obstacle against the use of bisphosphonates.

Key Words: Bisphosphonates · Bone diseases · Metabolic · Osteopenia

\section{INTRODUCTION}

Osteopenia is a condition in which bone mineral density (BMD) is low but not

Copyright (C) 2020 The Korean Society for Bone and Mineral Research

This is an Open Access article distributed under the terms of the Creative Commons Attribution Non-Commercial License (https://creativecommons.org/licenses/by-nc/4.0/) which permits unrestricted non-commercial use, distribution, and reproduction in any medium, provided the original work is properly cited.

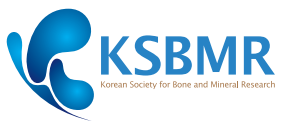
as low as osteoporosis.[1] For measurement of BMD, dual energy X-ray absorptiometry (DXA) of the hip and spine is currently the test of "gold standard".[2] According to the criteria of World Health Organization, osteopenia is diagnosed when T score is from -1 to -2.5 on DXA.[1]

Low BMD is a strong predictor of further development of osteoporosis and osteoporotic fractures.[3-5] People with osteopenia may have a higher risk of fractures.[5,6] In $82 \%$ of postmenopausal women with fractures showed T scores high- 
er than -2.5.[7]

Several studies have shown that anti-osteoporosis medication could decrease fracture risk and the economic burden of fracture in osteopenia patients.[8] But, pharmacological fracture prevention has been debated in osteopenia patients,[9] because of concerns about cost-effectiveness and adverse events including osteonecrosis of the jaw (ONJ) and atypical femoral fractures (AFFs) after long-term use of bisphosphonate.[9]

The recent the American College Physicians guidelines (2017) recommended that clinicians make the decision whether to treat osteopenic women 65 years of age or older who are at high risk for fracture based on a discussion of patient preference, fracture risk profile, and benefits, harms, and costs of medications.[10] Therefore, clinicians' attitude is important in the treatment of osteopenia. Nevertheless, there has been no study on this issue.

The purpose of our survey was to evaluate the clinicians' attitudes on the treatment of osteopenia by using a questionnaire.

\section{METHODS}

A survey was conducted by using a questionnaire during 3 osteoporosis-related symposia, which were held from August 2019 to September 2019 in South Korea.

The questionnaire was made referring to the 2017 American College of Physicians clinical practice guideline on the treatment of low bone density or osteoporosis to prevent fractures.[10]

The questionnaire was composed of 6 items: (1) do you think anti-osteoporosis medications are necessary in osteopenia patients?; (2) what is your preference to manage osteopenia patients (multiple choice), except for anti-osteoporosis medications?; (3) what is your indication for the anti-osteoporosis medication in osteopenia patients? (multiple choice); (4) what kind of anti-osteoporosis medication do you prefer to treat osteopenia patients? (multiple choice); (5) do you use bisphosphonates or not?; and (6) if not, what is the reason for not using bisphosphonates? (multiple choice; Table 1).

\section{Data analysis}

Categorical and dichotomous variables were summarized with frequencies and percentage.

\section{RESULTS}

A total of 180 physicians to this survey 173 (96.1\%) among them replied questionnaire. Specialties of the respondents were shown in Table 2.

\section{Necessity of anti-osteoporosis medications in osteopenia patients}

One hundred and fifty (86.7\%) replied that anti-osteoporosis medications were necessary in osteopenia patients and the remaining $23(13.3 \%)$ replied that anti-osteoporosis medications were not necessary in osteopenia patients. There was no significant difference based on the specialties of the respondents.

\section{Non-anti-osteoporosis medications or managements to treat osteopenia patients (multiple choice)}

One hundred and twenty-three (71.1\%) supplemented calcium and vitamin D, 95 (54.9\%) recommended regular exercise and lifestyle modification, and 55 (31.8\%) monitored BMDs of their patients.

\section{Indications for the anti-osteoporosis medication in osteopenia patients? (multiple choice)}

Indications for the anti-osteoporosis medication in osteopenia patients were past medical history of osteoporotic fracture in 85 (49.1\%), T-score between -2.5 and -1.0 on of DXA in 73 (42.2\%), previous history of osteoporosis in 44 (25.4\%), risk of osteoporotic fracture according to fracture risk assessment tool (FRAX ${ }^{\circledR}$ ) in 34 (19.7\%), progressive bone loss even with nutritional supplements in 31 (17.9\%).

\section{Options of anti-osteoporosis medications to treat osteopenia patients (multiple choice)}

One hundred and sixteen (67.1\%) preferred bisphosphonates; 87 (50.3\%) oral bisphosphonates and 29 (16.8\%) intravenous bisphosphonates, 93 (53.8\%) preferred selective estrogen receptor modulator (SERM), and 24 (13.9\%) preferred hormone replacement therapy (HRT).

\section{Use of bisphosphonates in osteopenia patients} One hundred and twenty-eight responders (74.0\%, 128/ 173) replied that they used bisphosphonate in osteopenia patients, 31 (17.9\%) responders replied that they did not 
Table 1. Questionnaire on the management of osteopenia patients

1. Where is your institute located at?

2. What is your specialty?
a. Orthopedics
b. Internal medicine
c. Gynecology
d. Neurosurgery
e. Family medicine
f. Rehabilitation medicine
g. Others (

3. Do you think anti-osteoporosis medications are necessary in osteopenia patients?
a. Yes
b. No

4. What is your current management to prevent fracture in patients with osteopenia except anti-osteoporosis medications (multiple answers allowed)?
a. Regular follow-up of bone mineral density
b. Calcium or vitamin $D$ supplements
c. Recommendation of exercise and lifestyle modification
d. Other (

5. What is your indication for the anti-osteoporosis medications in osteopenia patients (multiple answers allowed)?
a. Osteopenia diagnosed in DXA
b. Unsuccessful results with nutrient supplements or other non-medical treatments
c. Change from osteoporosis to osteopenia
d. History of osteoporotic fracture
e. High risk of osteoporotic fracture based on FRAX® assessment
f. Other (

6. What kind of anti-osteoporosis medication do you prefer to treat osteopenia patients (multiple answers allowed)?
a. Hormone replace therapy (estrogen, tibolone)
b. Selective estrogen receptor modulators (raloxifene, bazedoxifene single/combination drug)
c. Oral bisphosphonate (risedronate single/combination drug)
d. Intravenous bisphosphonate injection (zoledronate)
e. Other (

7. Do you use bisphosphonate use in osteopenia patients or not? (yes, no) If not, what is the reason for not using bisphosphonates (multiple answers allowed)?

a. Restriction by national health insurance

b. Bisphosphonate-related complications; atypical femoral fractures and bisphosphonate-related osteonecrosis of the jaw

c. Other (
DXA, dual energy X-ray absorptiometry; FRAX, fracture risk assessment tool.

Table 2. Specialties of 173 respondents

\begin{tabular}{lc}
\hline Specialty & $\mathrm{n}$ \\
\hline Orthopedic surgery & 68 \\
Internal medicine & 55 \\
Neurosurgery & 15 \\
Obstetrics and gynecology & 14 \\
Family medicine & 12 \\
Rehabilitation & 3 \\
Others & 6 \\
\hline
\end{tabular}

use and the remaining 14 (8.1\%) responders did not answer (Fig. 1).

\section{Reason for not using bisphosphonates} In the 31 respondents, who did not use bisphosphonates, reasons for not using were (1) restricted reimbursement by national health insurance in 24 (77.4\%, 24/31) and (2) bisphosphonate-related complications; AFFs and bisphosphonate-related ONJ, in 19 (61.3\%, 19/31) (Fig. 2). We allowed the respondents to submit any other reason through 


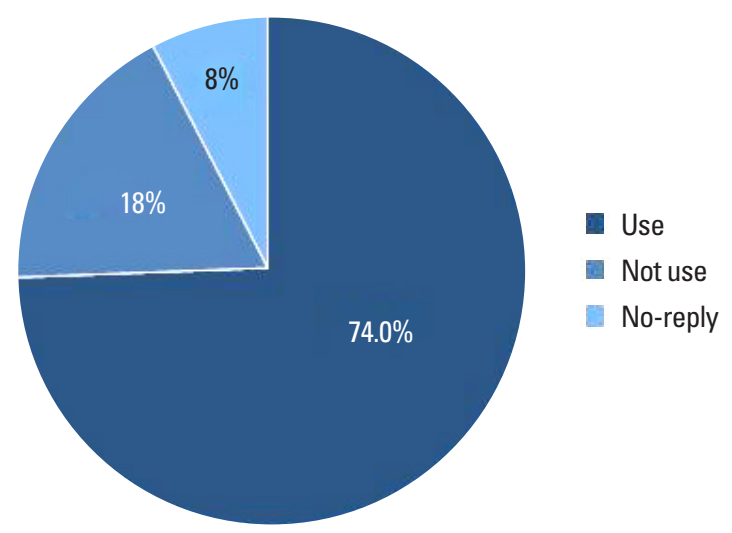

Fig. 1. Physicians' attitudes on prescribing bisphosphonate for osteopenia treatment.

the open-ended text box; however, there was no reply submitted.

\section{DISCUSSION}

Reportedly, the prevalence of osteopenia among the population aged 50 years and older was $46.5 \%$ in males and $48.7 \%$ in females in South Korea.[11] Our survey figured out the attitudes of South Korean physicians to manage osteopenia patients.

In this survey, most physicians (86.7\%) advocated the use of anti-osteoporosis medications were necessary in osteopenia patients to prevent fracture. The most common indication for the use of anti-osteoporosis medication in osteopenia patients was a history of osteoporotic fracture.

In May 2015, South Korean health insurance started to reimburse the prescription of anti-osteoporosis medication for patients with osteoporotic fractures regardless of T-score.[12] This change of reimbursement guideline might explain the high advocating rate of anti-osteoporosis medications in our survey.

The use of FRAX ${ }^{\circledR}$ has been popularized to guide the preventive treatment of patients at risk of osteoporotic fractures (https://www.sheffield.ac.uk/FRAX/). However, the $F R A X^{\circledR}$ is not reimbursed in South Korea, yet, and only $19.7 \%$ of our responders indicated the use FRAX ${ }^{\circledast}$ for the anti-osteoporosis medication.

Bisphosphonates and SERM have been reported to reduce fracture risk in osteopenia patients.[13-15] HRT has been known to prevent or reverse bone loss in postmenopausal women.[16] In our study, the most preferred medi-

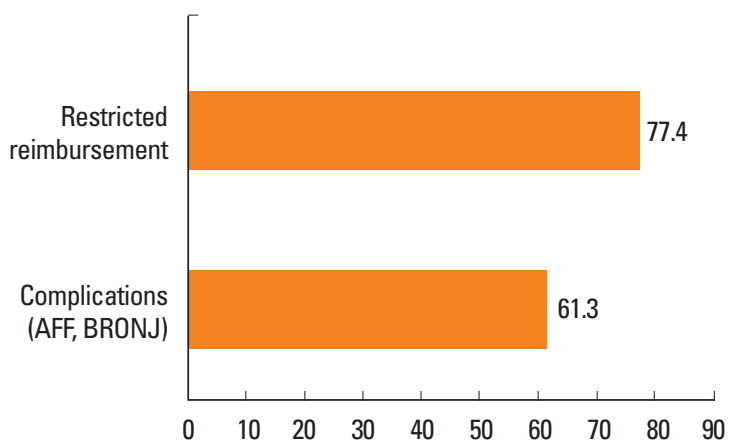

Fig. 2. The reason for not using bisphosphonate in osteopenia patients.

cation for osteopenia patients was bisphosphonates, followed by SERM and HRT.

Most responders (74.0\%) prescribed bisphosphonates, while $17.9 \%$ of the responders did not prescribe bisphosphonates in osteopenia patients.

Major reason $(77.4 \%, 24 / 31)$ for not prescribing bisphosphonates was restricted reimbursement from the health insurance. The reimbursement issue was a serious obstacle to osteoporosis treatment in Asia-Pacific region as well as in the United States.[17,18] In May 2015, the Korean Health Insurance Review and Assessment Service (HIRA) permitted the preventive use of osteoporosis medications in osteopenia patients, when they had a history of osteoporotic fracture.[12] Since October 2018, the HIRA has reimbursed the preventive use of bisphosphonates for patients who took glucocorticoids for more than 3 months.[19] The second common reason $(61.3 \%, 19 / 31)$ for not using bisphosphonates was a concern of AFFs and bisphosphonate-related ONJ. However, recent studies have demonstrated that the benefit of the bisphosphonate treatment exceeded the risk of adverse events.[20,21] The HRT was unfavored probably due to the potential risk of breast cancer.[22]

This study has limitations. This survey involved small number (173) of physicians, and our results might not represent the general opinion of physicians in South Korea. Furthermore, due to privacy issues, the age and gender of respondents were not analyzed, which could also be confounding factors.

\section{CONCLUSIONS}

Most South Korean physicians (86.7\%) thought anti-osteoporosis medications were necessary in osteopenia pa- 
tients. They favored bisphosphonates, SERM, and HRT for the treatment of osteopenia patients to prevent fractures. Restricted reimbursement by the national health insurance was the major obstacle against the use of bisphosphonates.

\section{DECLARATIONS}

\section{Conflict of interest}

No potential conflict of interest relevant to this article was reported.

\section{ORCID}

Joo Hyung $\mathrm{Ha}$ https://orcid.org/0000-0001-9163-3177 Hong Seok Kim https://orcid.org/0000-0002-9524-7019 Young-Kyun Lee https://orcid.org/0000-0001-6564-4294 Kyung-Hoi Koo https://orcid.org/0000-0001-5251-2911

\section{REFERENCES}

1. Zhang J, Morgan SL, Saag KG. Osteopenia: debates and dilemmas. Curr Rheumatol Rep 2013;15:384.

2. Black DM, Cummings SR, Genant HK, et al. Axial and appendicular bone density predict fractures in older women. J Bone Miner Res 1992;7:633-8.

3. Gourlay ML, Fine JP, Preisser JS, et al. Bone-density testing interval and transition to osteoporosis in older women. $\mathrm{N}$ Engl J Med 2012;366:225-33.

4. Hillier TA, Cauley JA, Rizzo JH, et al. WHO absolute fracture risk models (FRAX): do clinical risk factors improve fracture prediction in older women without osteoporosis? J Bone Miner Res 2011;26:1774-82.

5. Cranney A, Jamal SA, Tsang JF, et al. Low bone mineral density and fracture burden in postmenopausal women. CMAJ 2007;177:575-80.

6. Kanis JA. Diagnosis of osteoporosis. Osteoporos Int 1997;7 Suppl 3:S108-16.

7. Siris ES, Chen YT, Abbott TA, et al. Bone mineral density thresholds for pharmacological intervention to prevent fractures. Arch Intern Med 2004;164:1108-12.

8. Kim HY, Ha YC, Kim TY, et al. Healthcare costs of osteoporotic fracture in Korea: Information from the national health insurance claims database, 2008-2011. J Bone Metab 2017; 24:125-33.

9. Zhang J, Saag KG, Curtis JR. Long-term safety concerns of antiresorptive therapy. Rheum Dis Clin North Am 2011;37: 387-400.

10. Qaseem A, Forciea MA, McLean RM, et al. Treatment of low bone density or osteoporosis to prevent fractures in men and women: A clinical practice guideline update from the American college of physicians. Ann Intern Med 2017; 166:818-39.

11. Park EJ, Joo IW, Jang MJ, et al. Prevalence of osteoporosis in the Korean population based on Korea National Health and Nutrition Examination Survey (KNHANES), 2008-2011. Yonsei Med J 2014;55:1049-57.

12. Yoo JH, Moon SH, Ha YC, et al. Osteoporotic fracture: 2015 position statement of the Korean society for bone and mineral research. J Bone Metab 2015;22:175-81.

13. Eriksen EF. Treatment of osteopenia. Rev Endocr Metab Disord 2012;13:209-23.

14. Gu JM, Wang L, Lin $\mathrm{H}$, et al. The efficacy and safety of weekly 35-mg risedronate dosing regimen for Chinese postmenopausal women with osteoporosis or osteopenia: 1-year data. Acta Pharmacol Sin 2015;36:841-6.

15. Reid IR, Horne AM, Mihov B, et al. Fracture prevention with zoledronate in older women with osteopenia. N Engl J Med 2018;379:2407-16.

16. Gallagher JC. Moderation of the daily dose of HRT: prevention of osteoporosis. Maturitas 1999;33 Suppl 1:S57-63.

17. Korthoewer D, Chandran M. Osteoporosis management and the utilization of $F R A X^{\circledR}$ : a survey amongst health care professionals of the Asia-Pacific. Arch Osteoporos 2012;7:193-200.

18. Ha YC, Lee YK, Lim YT, et al. Physicians' attitudes to contemporary issues on osteoporosis management in Korea. J Bone Metab 2014;21:143-9.

19. Park SY, Gong HS, Kim KM, et al. Korean guideline for the prevention and treatment of glucocorticoid-induced osteoporosis. J Bone Metab 2018;25:195-211.

20. Ilyas Z, Camacho PM. Rare adverse effects of bisphosphonate therapy. Curr Opin Endocrinol Diabetes Obes 2019; 26:335-8.

21. Won SH, Ahn S, Ji E, et al. Hip and subtrochanteric or femoral shaft fractures after bisphosphonate use in Korean women, using Korean national sample cohort. J Korean Med Sci 2020;35:e193.

22. Compston JE. The risks and benefits of HRT. J Musculoskelet Neuronal Interact 2004;4:187-90. 
